\title{
Incidence of postoperative suprachoroidal hemorrhage after glaucoma filtration surgeries in the United States
}

This article was published in the following Dove Press journal:

Clinical Ophthalmology

2 April 2015

Number of times this article has been viewed

\author{
Kamyar Vaziri' \\ Stephen G Schwartz' \\ Krishna S Kishor' \\ Jorge A Fortun' \\ Darius M Moshfeghi \\ Andrew A Moshfeghi ${ }^{3}$ \\ Harry W Flynn Jr' \\ 'Department of Ophthalmology, \\ Bascom Palmer Eye Institute, \\ University of Miami Miller School \\ of Medicine, Miami, FL, USA; \\ ${ }^{2}$ Department of Ophthalmology, Byers \\ Eye Institute, Stanford University \\ School of Medicine, Palo Alto, CA, \\ USA; ${ }^{3}$ Department of Ophthalmology, \\ USC Eye Institute, University of \\ Southern California Keck School \\ of Medicine, Los Angeles, CA, USA
}

Purpose: To report the 3-month incidence rates of postoperative suprachoroidal hemorrhage after glaucoma filtration surgeries and to examine the subsequent surgical treatments in these eyes. Methods: This is a retrospective study using the nationally pooled, insurance claim-based MarketScan databases from the years 2007-2011. Patients with records of trabeculectomy and/ or tube shunt procedures were identified, and all cases of "definite" (ie, properly coded) and "suspected" (possibly miscoded) postoperative suprachoroidal hemorrhage occurring within 3 months of their glaucoma filtration procedures were captured along with the surgical interventions used for this condition. Kaplan-Meier survival analysis was used to evaluate the 3-month incidence rates of suprachoroidal hemorrhage, and regression analysis was applied to calculate the odds ratios, confidence intervals, and $P$-values.

Results: There were 17,843 trabeculectomies and 9,597 tube shunt surgeries identified. Among these, there were 107 cases (247 including "suspected" cases) of postoperative suprachoroidal hemorrhage within 3 months of trabeculectomy and 113 cases (255 including "suspected" cases) within 3 months of tube shunt procedures. The 3-month cumulative incidence rate of postoperative suprachoroidal hemorrhage ranged from $0.6 \% \pm 0.06 \%$ to $1.4 \% \pm 0.09 \%$ after trabeculectomy and $1.2 \% \pm 0.11 \%$ to $2.7 \% \pm 0.16 \%$ after tube shunt surgery. Postoperative suprachoroidal hemorrhage was almost twice as likely to occur after tube shunt surgeries than after trabeculectomies for both "definite" and "definite" plus "suspected" cases (odds ratio, 1.98; 95\% confidence interval, 1.51-2.58; $P<0.001$; and odds ratio, $1.95 ; 95 \%$ confidence interval, $1.63-2.32 ; P<0.001$, respectively). Among the 502 "definite" and "suspected" cases of postoperative suprachoroidal hemorrhage, $32.9 \%$ (165 cases) had a treatment record of choroidal tap and $8.8 \%$ (44 cases) had a treatment record of pars plana vitrectomy.

Conclusion: In this sample, the 3-month cumulative incidence rate of postoperative suprachoroidal hemorrhage was $0.6 \%-1.4 \%$ after trabeculectomy and $1.2 \%-2.7 \%$ after tube shunt procedures, and the majority of the cases appeared to be managed without further surgery. Postoperative suprachoroidal hemorrhage was almost twice as likely to occur after tube shunt surgeries as after trabeculectomies.

Keywords: suprachoroidal hemorrhage, choroidal effusion, trabeculectomy, tube shunt, choroidal tap, pars plana vitrectomy

\section{Introduction}

Suprachoroidal hemorrhage (Figures 1 and 2) is an uncommon but potentially vision-threatening event ${ }^{1}$ that may occur spontaneously, ${ }^{2-4}$ after trauma, ${ }^{5-7}$ or as a complication of ocular procedures including cataract surgery, secondary lens implantation, pars plana vitrectomy (PPV), scleral buckling, penetrating keratoplasty, and glaucoma surgeries. ${ }^{5,7-12}$
Bascom Palmer Eye Institute, University of Miami Miller School of Medicine,

3II 9th Street North, \#I00, Naples, FL

34102 , USA

Tel + I 2396593937

Fax +l 2396593982

Email sschwartz2@med.miami.edu 


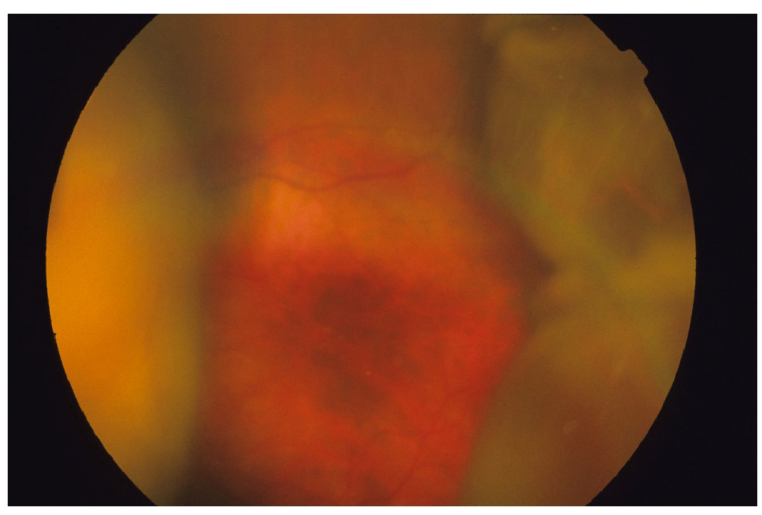

Figure I Fundus photography, left eye, demonstrating an example of nonappositional suprachoroidal hemorrhage that obscures the view of the optic disc.

The pathophysiology of nontraumatic suprachoroidal hemorrhage is unknown, but it is suspected to involve the rupture of a short or long posterior ciliary artery, ${ }^{13,14}$ either directly or secondary to hypotony. ${ }^{15,16}$

The reported incidence rates of postoperative suprachoroidal hemorrhage after glaucoma surgeries vary from $0.7 \%$ to $6.1 \% .^{10-12,17-21}$ Numerous potential risk factors have been associated with the development of intraoperative and postoperative suprachoroidal hemorrhage, and the ones pertaining to glaucoma surgeries include high preoperative intraocular pressure (IOP), severe postoperative hypotony, aphakia, pseudophakia, anticoagulation, white race, prior intraocular surgery, low postoperative IOP, systemic hypertension, ischemic heart disease, and pulmonary disease. ${ }^{11,22,23}$

We used a large nationwide insurance claim-based database to assess the 3-month cumulative incidence rate and recorded surgical treatments of postoperative suprachoroidal hemorrhage after trabeculectomy and tube shunt procedures.

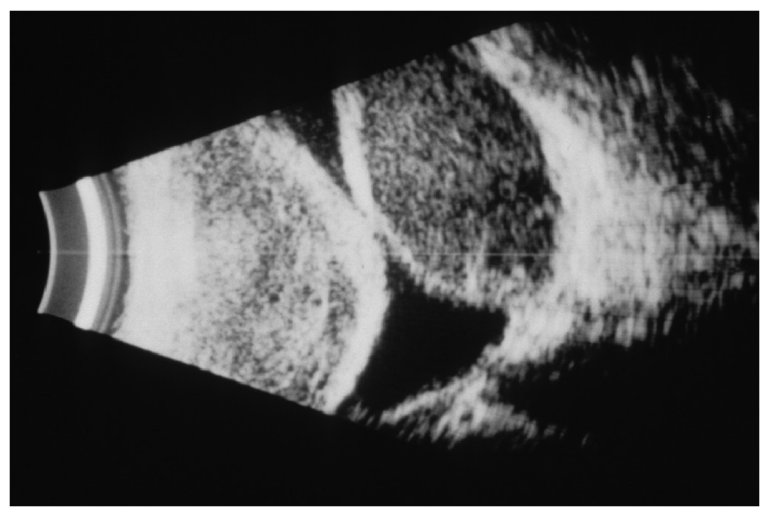

Figure 2 B-scan echography demonstrating an example of appositional suprachoroidal hemorrhage.

\section{Methods \\ Data source}

This is a retrospective cross-sectional case series using the MarketScan commercial Claims and Encounters and Medicare Supplemental and Coordination of Benefit Outpatient Services and inpatient databases (Truven Health Analytics, Ann Arbor, MI, USA) from 2007 to 2011 (the most recent year the database was available to us). The MarketScan family of databases comprises the largest conveniencebased proprietary database in the United States, annually encompassing approximately 40-50 million patients with employer-sponsored or supplemental insurance. These databases consist of deidentified, individual-level health records (inpatient and outpatient) obtained from large employers, hospitals, and Medicare programs. The MarketScan database contains deidentified and anonymized records and complies with the privacy requirements of the Health Information Portability and Accountability Act of 1996 (HIPPA); institutional review board approval was not required.

The MarketScan Outpatient Services databases include demographic, provider type, and insurance information along with up to four diagnosis codes and one procedure code per recorded insurance claim (coded using the International Classification of Diseases, Ninth Revision [ICD-9]; and Current Procedural Terminology [CPT], respectively). Further details regarding the structure and HIPPA compliance of the MarketScan databases can be found elsewhere. ${ }^{24}$ We have previously used these databases to study the incidence of endophthalmitis after trabeculectomy. ${ }^{25}$

\section{Study sample and statistical analysis}

We used CPT codes to select all the patients with records of trabeculectomy (CPT codes 66170, 66172) and tube shunt surgery (CPT codes 66180, 0191T, and 0192T). To calculate 3-month incidence rates of postoperative suprachoroidal hemorrhage, only patients with 3 months of continuous medical insurance after their surgery date were included. (Using this technique, we were unable to record cases of intraoperative suprachoroidal hemorrhage.) Suprachoroidal hemorrhage diagnosis among these patients was queried using ICD-9 codes 363.61, 363.62, 363.63, and 363.72. In our analysis, these were considered "definite" cases of postoperative suprachoroidal hemorrhage. To account for the cases of postoperative suprachoroidal hemorrhage that may have been miscoded as choroidal effusion, patients with a diagnosis of choroidal effusion (ICD-9 codes 363.70 and 363.71) were also selected, and these were considered "suspected" cases of postoperative suprachoroidal hemorrhage. In patients with 
a record of both suprachoroidal hemorrhage and choroidal effusion within 3 months of the same surgery, their choroidal effusion event was excluded from the analysis.

To capture the surgical treatments recorded for the queried cases of postoperative suprachoroidal hemorrhage, cases of PPV (CPT codes 67036, 67038, 67039, 67040, 67041, 67042, 67043) and choroidal drainage (recorded under CPT code 67015, "Aspiration or release of vitreous, subretinal or choroidal fluid, pars plana approach [posterior sclerotomy]") accompanied with one of the suprachoroidal hemorrhage or effusion ICD-9 codes were identified. We also queried CPT code 67105 ("repair of retinal detachment, one or more sessions; photocoagulation, with or without drainage of subretinal fluid") because the codes 67015 and 67105 may be easy to confuse.

We used descriptive statistics and cross-tabulations to evaluate prevalence estimates of comorbidities and demographic information. Kaplan-Meier survival analysis was used to calculate 3-month cumulative incidence of suprachoroidal hemorrhage after glaucoma filtering surgeries. Regression analysis was used to calculate the odds ratio, confidence intervals, and $P$-value. A $P$-value of $<0.05$ was considered to be statistically significant. All statistical analyses were performed with SPSS (version 22; SPSS Inc., Chicago, IL, USA).

\section{Results}

In the databases, there were a total of 27,440 glaucoma filtration procedures, including 17,843 trabeculectomies and 9,597 tube shunt surgeries, among 21,523 patients who had 3 months of continuous health insurance after their procedure date. The age of patients with glaucoma filtration records ranged from infancy ( $<1$ year old) to 99 years old, with a mean age of $66.4 \pm 12.8$ years. There were 13,529 males (49.3\%) and 13,911 females (50.7\%). There were 107 cases of "definite" postoperative suprachoroidal hemorrhage within 3 months of trabeculectomy and 113 cases within 3 months of tube shunt (total of 220 cases). Including only the "definite" cases of postoperative suprachoroidal hemorrhage,
Kaplan-Meier analysis showed the 3-month cumulative incidence rate of suprachoroidal hemorrhage to be $0.6 \% \pm 0.06 \%$ after trabeculectomy and $1.2 \% \pm 0.11 \%$ after tube shunt, and there was an overall rate of $0.8 \% \pm 0.05 \%$ after both types of surgeries (Table 1).

In addition to the "definite" cases of postoperative suprachoroidal hemorrhage, there were an additional (possibly miscoded) 170 "suspected" cases after trabeculectomy. In 30 instances, there were records of both suprachoroidal hemorrhage and choroidal effusion within 3 months of the same surgery in which only the suprachoroidal hemorrhage event was counted, bringing up the total to 247 cases of "definite" and "suspected" cases of postoperative suprachoroidal hemorrhage, leading to a 3-month cumulative incidence rate of $1.4 \% \pm 0.09 \%$ after trabeculectomy (Table 1 ).

Similarly, the "suspected" cases of postoperative suprachoroidal hemorrhage after tube shunt $(\mathrm{N}=171)$ were added to the definite cases (total, $\mathrm{N}=255$; the 29 duplicate instances of both suprachoroidal hemorrhage and effusion after the same procedure were counted as the same event). In this scenario, the 3-month cumulative incidence rate of suprachoroidal hemorrhage after tube shunt surgery was $2.7 \% \pm 0.16 \%$. Overall, after inclusion of both "suspected" and "definite" cases, 3-month cumulative rate of suprachoroidal hemorrhage after both trabeculectomy and tube shunt surgery $(\mathrm{N}=502)$ was $1.8 \% \pm 0.08 \%$ (Table 1 ).

Using only the "definite" cases of postoperative suprachoroidal hemorrhage, this complication was twice as likely to occur after tube shunt as after trabeculectomy (odds ratio, 1.98; 95\% confidence interval, $1.51-2.58 ; P<0.001)$. The inclusion of "suspected" cases of suprachoroidal hemorrhage yielded very similar increased odds after tube shunt surgery (OR, 1.95; 95\% confidence interval, 1.63-2.32; $P<0.001)$.

In another analysis, surgical treatments (PPV or choroidal tap) for the treatment of suprachoroidal hemorrhage were captured. Among the 502 "definite" and "suspected" cases, 165 cases (32.9\%) had a record of choroidal tap (CPT code

Table I Suprachoroidal hemorrhage cases after glaucoma surgeries

\begin{tabular}{|c|c|c|c|c|c|}
\hline Event & Definite cases & Suspected cases & Total & $\begin{array}{l}\text { 3-month incidence } \\
\text { rate of definite cases }\end{array}$ & $\begin{array}{l}\text { 3-month incidence rate of } \\
\text { definite plus suspected cases }\end{array}$ \\
\hline $\begin{array}{l}\text { Suprachoroidal hemorrhage after } \\
\text { trabeculectomy procedures }\end{array}$ & 107 & 170 & 247 & $0.6 \%+0.06 \%$ & $1.4 \%+0.09 \%$ \\
\hline $\begin{array}{l}\text { Suprachoroidal hemorrhage after tube } \\
\text { shunt procedures }\end{array}$ & 113 & $|7|$ & 255 & $1.2 \%+0.11 \%$ & $2.7 \%+0.16 \%$ \\
\hline $\begin{array}{l}\text { Suprachoroidal hemorrhage after both } \\
\text { trabeculectomy and tube shunt procedures }\end{array}$ & 220 & 341 & 502 & $0.8 \%+0.05 \%$ & $1.8 \%+0.08 \%$ \\
\hline
\end{tabular}


67015) as a treatment (110 of the "definite" and 55 of the "suspected" cases), and 44 (8.8\%) had PPV (CPT codes 67036, 67038, 67039, 67040, 67041, 67042, and 67043) recorded as their treatment (30 of the "definite" postoperative suprachoroidal hemorrhage cases and 14 of the "suspected" cases). In addition, there were five cases (three "definite" and two "suspected") that were treated using CPT code 67105 ("Repair of retinal detachment"), rather than choroidal tap (CPT code 67015). Two of these five cases also had a diagnosis of retinal detachment (ICD-9 code 361.05; "Recent retinal detachment, total or subtotal"), whereas the other three had no records of retinal detachment.

\section{Discussion}

Suprachoroidal hemorrhage is often associated with unfavorable visual outcomes even with appropriate surgical intervention, especially when the hemorrhage involves all four quadrants or is associated with retinal detachment. One study reported that only $34 \%$ of eyes with suprachoroidal hemorrhage achieved a final visual acuity of $20 / 200,{ }^{5}$ and another study reported that $17 \%$ of patients with suprachoroidal hemorrhage ended up with a final visual acuity of no light perception. ${ }^{26}$

The reported incidence rates of suprachoroidal hemorrhage after glaucoma procedures vary from study to study. This variation might be attributed to the smaller sample sizes of the majority of these reports (ranging from 197 to 2,752 analyzed glaucoma procedures). ${ }^{21,23}$ In our analysis, we identified 17,843 trabeculectomies and 9,597 tube shunt surgeries, for a total of 27,440 surgical procedures, and found that 3-month cumulative incidence rate of suprachoroidal hemorrhage was $0.6 \%-1.4 \%$ after trabeculectomy and $1.2 \%-2.7 \%$ after tube shunt. Previous reports have shown higher rates of suprachoroidal hemorrhage when antimetabolites (5-fluorouracil or mitomycin-C) were used. ${ }^{11,27}$ The reason was attributed to the lower IOPs achieved by using antimetabolites, ${ }^{27}$ which is supported by the findings of low postoperative IOPs as a risk factor for the development of suprachoroidal hemorrhage. ${ }^{23}$ As the use of antimetabolites is not coded separately on medical insurance claims, we were unable to evaluate the proportion of trabeculectomies in which antimetabolites were used in these databases.

In the current study, postoperative suprachoroidal hemorrhage was almost two times more likely to occur after tube shunt than after trabeculectomy. This is similar to the findings of Tuli et al and Jeganathan et al who also found that this condition was more than three times more likely to occur after tube shunt surgeries. ${ }^{11,23}$ The hypothesized explanation has been that patients requiring drainage devices may have higher preoperative IOP, leading to a larger drop in the IOP postoperatively. ${ }^{11,23}$

The present study analyzed only trabeculectomies and tube shunt surgeries and did not include nonpenetrating glaucoma surgeries, including deep sclerectomies, viscocanalostomies, and canaloplasties. A recent systematic review and meta-analysis reported that nonpenetrating glaucoma surgeries were less effective in lowering IOP but also had lower risks for choroidal effusion and other complications, although suprachoroidal hemorrhage was not specifically reported. ${ }^{28}$

There are few published guidelines regarding the treatment of postoperative suprachoroidal hemorrhage. Its management is typically at the discretion of the physician. Whether earlier surgical intervention (either choroidal tap or PPV) offers better visual outcomes over conservative management remains unclear. Although several authors have advocated earlier surgical management, ${ }^{29-31}$ others have not shown a clear advantage of surgical intervention over conservative management, especially in the cases of limited suprachoroidal hemorrhage..$^{15,20,23}$ In our results, approximately 33\% of suprachoroidal hemorrhage cases were treated with choroidal tap, and approximately $9 \%$ were treated with PPV, suggesting the majority of the cases were managed conservatively. Among the five cases of "definite" and "suspected" suprachoroidal hemorrhage that were treated with CPT code 67105 (repair of retinal detachment), we suspect three were miscoded, as they had no records of retinal detachment, whereas the other two either had retinal detachment alone or had retinal detachment and suprachoroidal hemorrhage/effusion.

The current study used a nationally pooled database containing data from all 50 states, yielding large numbers of glaucoma surgeries. This nationwide database also allowed us to avoid the potential biases associated with singlecentered and surgeon-specific data. As for limitations, as the MarketScan databases are insurance claim-based and the medical diagnoses and surgical procedures are recorded as ICD-9 and CPT codes, respectively, the accuracy of the data depends on proper coding. For this reason, we also included the cases of choroidal effusions as "suspected" cases of suprachoroidal hemorrhage and presented the incidence rates as a range encompassing both "definite" cases of postoperative suprachoroidal hemorrhage and "definite" plus "suspected" cases.

In addition, the MarketScan databases provide only ICD-9 diagnostic and CPT procedural codes and do not 
provide detailed clinical information about individual patients, such as individual IOP measurements, the severity of the suprachoroidal hemorrhages, surgical details, and visual outcomes. Therefore, we could not provide this information in the present study.

In conclusion, the 3-month cumulative incidence rate of postoperative suprachoroidal hemorrhage was $0.6 \%-1.4 \%$ after trabeculectomy and $1.2 \%-2.7 \%$ after tube shunt procedures. This condition was two times more likely to occur after tube shunt surgery than after trabeculectomy. The majority of suprachoroidal hemorrhage cases did not have any recorded surgical managements, which might imply most were treated without additional surgery. Of course, these incidence rates were calculated from a set of insurance databases and may not be generally applicable to all clinical situations. Future prospective studies are required to reevaluate the prevalence of different treatment modalities in the management of postoperative suprachoroidal hemorrhage.

\section{Disclosure}

The authors from the University of Miami are partially supported by the National Institutes of Health Center Core Grant P30EY014801 and an unrestricted grant from Research to Prevent Blindness, New York City. SGS has received consulting fees from Alimera and Bausch + Lomb, speakers' fees from ThromboGenics, and writers' fees from Vindico. The other authors report no conflicts of interest in this work.

\section{References}

1. Chu TG, Green RL. Suprachoroidal hemorrhage. Surv Ophthalmol. 1999;43(6):471-486.

2. Yang SS, Fu AD, McDonald HR, Johnson RN, Ai E, Jumper JM. Massive spontaneous choroidal hemorrhage. Retina. 2003;23(2):139-144.

3. Hammam T, Madhavan C. Spontaneous suprachoroidal haemorrhage following a valsalva manoeuvre. Eye (Lond). 2003;17(2):261-262.

4. Nguyen HN, Nork TM. Massive spontaneous suprachoroidal hemorrhage in a young woman with cystic fibrosis and diabetes mellitus on anticoagulants. Retin Cases Brief Rep. 2012;6(2):216-218.

5. Reynolds MG, Haimovici R, Flynn HW Jr, DiBernardo C, Byrne SF, Feuer W. Suprachoroidal hemorrhage. Clinical features and results of secondary surgical management. Ophthalmology. 1993;100(4):460-465.

6. Dong X, Yuan G, Wang W. [Surgical treatment of traumatic suprachoroidal hemorrhage]. Zhonghua Yan Ke Za Zhi. 2002;38(11):654-656.

7. Jin W, Xing Y, Xu Y, Wang W, Yang A. Management of delayed suprachoriodal haemorrhage after intraocular surgery and trauma. Graefes Arch Clin Exp Ophthalmol. 2014;252(8):1189-1193.

8. Duncker GI, Rochels R. Delayed suprachoroidal hemorrhage after penetrating keratoplasty. Int Ophthalmol. 1995-1996;19(3):173-176.

9. Wang LC, Yang CM, Yang CH, et al. Clinical characteristics and visual outcome of non-traumatic suprachoroidal haemorrhage in Taiwan. Acta Ophthalmol (Copenh). 2008;86(8):908-912.

10. Jampel HD, Musch DC, Gillespie BW, Lichter PR, Wright MM, Guire KE; Collaborative Initial Glaucoma Treatment Study Group. Perioperative complications of trabeculectomy in the collaborative initial glaucoma treatment study (CIGTS). Am J Ophthalmol. 2005;140(1):16-22.
11. Tuli SS, WuDunn D, Ciulla TA, Cantor LB. Delayed suprachoroidal hemorrhage after glaucoma filtration procedures. Ophthalmology. 2001;108(10):1808-1811.

12. Gedde SJ, Herndon LW, Brandt JD, Budenz DL, Feuer WJ, Schiffman JC; Tube Versus Trabeculectomy Study Group. Postoperative complications in the Tube Versus Trabeculectomy (TVT) study during five years of follow-up. Am J Ophthalmol. 2012;153(5):804-814.e1.

13. Manschot WA. The pathology of expulsive hemorrhage. Am J Ophthalmol. 1955;40(1):15-24.

14. Pe'er J, Weiner A, Vidaurri L. Clinicopathologic report of spontaneous expulsive hemorrhage. Ann Ophthalmol. 1987;19(4):139-141.

15. Gressel MG, Parrish RK II, Heuer DK. Delayed nonexpulsive suprachoroidal hemorrhage. Arch Ophthalmol. 1984;102(12):1757-1760.

16. Maumenee AE, Schwartz MF. Acute intraoperative choroidal effusion. Am J Ophthalmol. 1985;100(1):147-154.

17. Al-Mobarak F, Khan AO. Two-year survival of Ahmed valve implantation in the first 2 years of life with and without intraoperative mitomycin-C. Ophthalmology. 2009;116(10):1862-1865.

18. Christakis PG, Tsai JC, Kalenak JW, et al. The Ahmed versus Baerveldt study: three-year treatment outcomes. Ophthalmology. 2013;120(11):2232-2240.

19. Givens K, Shields MB. Suprachoroidal hemorrhage after glaucoma filtering surgery. Am J Ophthalmol. 1987;103(5):689-694.

20. Ruderman JM, Harbin TS Jr, Campbell DG. Postoperative suprachoroidal hemorrhage following filtration procedures. Arch Ophthalmol. 1986;104(2):201-205.

21. Paysse E, Lee PP, Lloyd MA, et al. Suprachoroidal hemorrhage after Molteno implantation. J Glaucoma. 1996;5(3):170-175.

22. The Fluorouracil Filtering Surgery Study Group. Risk factors for suprachoroidal hemorrhage after filtering surgery. Am J Ophthalmol. 1992;113(5):501-507.

23. Jeganathan VSE, Ghosh S, Ruddle JB, Gupta V, Coote MA, Crowston JG. Risk factors for delayed suprachoroidal haemorrhage following glaucoma surgery. Br J Ophthalmol. 2008;92(10):1393-1396.

24. Danielson E. Health Research Data for the Real World: The MarketScan ${ }^{\circledR}$ Databases. Ann Arbor, MI: Truven Health Analytics; 2014. Available from: http://truvenhealth.com/Portals/0/Users/031/31/31/ PH_13434\%200314_MarketScan_WP_web.pdf. Accessed December 23, 2014.

25. Vaziri K, Kishor K, Schwartz SG, et al. Incidence of Bleb-Associated Endophthalmitis in the United States. J Clin Ophthalmol. 2015;9: 317-322.

26. Wirostko WJ, Han DP, Mieler WF, Pulido JS, Connor TB Jr, Kuhn E. Suprachoroidal hemorrhage: outcome of surgical management according to hemorrhage severity. Ophthalmology. 1998;105(12):2271-2275.

27. Ghadhfan FE, Khan AO. Delayed suprachoroidal hemorrhage after pediatric glaucoma surgery. J AAPOS. 2009;13(3):283-286.

28. Rulli E, Biagioli E, Riva I, et al. Efficacy and safety of trabeculectomy vs nonpenetrating surgical procedures: a systematic review and metaanalysis. JAMA Ophthalmol. 2013;131(12):1573-1582.

29. Abrams GW, Thomas MA, Williams GA, Burton TC. Management of postoperative suprachoroidal hemorrhage with continuous-infusion air pump. Arch Ophthalmol. 1986;104(10):1455-1458.

30. Ariano ML, Ball SF. Delayed nonexpulsive suprachoroidal hemorrhage after trabeculectomy. Ophthalmic Surg. 1987;18(9):661-666.

31. Pakravan M, Yazdani S, Afroozifar M, Kouhestani N, Ghassami M, Shahshahan M. An alternative approach for management of delayed suprachoroidal hemorrhage after glaucoma procedures. J Glaucoma. 2014;23(1):37-40. 


\section{Publish your work in this journal}

Clinical Ophthalmology is an international, peer-reviewed journal covering all subspecialties within ophthalmology. Key topics include: Optometry; Visual science; Pharmacology and drug therapy in eye diseases; Basic Sciences; Primary and Secondary eye care; Patient Safety and Quality of Care Improvements. This journal is indexed on

Submit your manuscript here: http://www.dovepress.com/clinical-ophthalmology-journal
PubMed Central and CAS, and is the official journal of The Society of Clinical Ophthalmology (SCO). The manuscript management system is completely online and includes a very quick and fair peer-review system, which is all easy to use. Visit http://www.dovepress.com/ testimonials.php to read real quotes from published authors. 